\title{
Infinitely many sign-changing solutions for a Schrödinger equation
}

\author{
Aixia Qian
}

Correspondence: qaixia@amss.ac.cn School of Mathematic Sciences, Qufu Normal University, Qufu Shandong, 273165, P. R. China

\section{Abstract}

We study a superlinear Schrödinger equation in the whole Euclidean space $\mathbb{R}^{n}$. By using a suitable sign-changing critical point, we prove that the problem admits infinitely many sign-changing solutions, under weaker conditions.

Keywords: Schrödinger equation, sign-changing critical point, (W*-PS) condition

\section{Introduction}

In this paper, we consider the following Schrödinger equation,

$$
\left\{\begin{array}{l}
-\Delta u+V(x) u=f(x, u), x \in \mathbb{R}^{N} \\
u(x) \rightarrow 0,|x| \rightarrow \infty
\end{array}\right.
$$

In order to overcome the lack of compactness of the problem, we assume that the potential $V(x)$ has a "good" behavior at infinity, in such a way the Schrödinger operator $-\Delta+V(x)$ on $L^{2}\left(\mathbb{R}^{N}\right)$ has a discrete spectrum. More precisely, we suppose $\left(V_{1}\right) V \in L_{\text {loc }}^{2}\left(\mathbb{R}^{N}\right), V$ is bounded from below;

$\left(V_{2}\right)$ There exists $r_{0}>0$ such that for any $h>0$

$$
\operatorname{meas}\left(B_{r_{0}}(y) \cap V_{h}\right) \rightarrow 0, \quad|y| \rightarrow+\infty,
$$

where meas $(A)$ denotes the Lebesgue measure of $A$ on $\mathbb{R}^{N}, B_{r_{0}}(\gamma)$ is the ball centered at $y$ with radius $r_{0}$ and $V^{h}=\left\{x \in \mathbb{R}^{N}: V(x)<h\right\}$.

Of course, $V(x)$ above can satisfy the condition $\left(S_{1}\right)$ or $\left(\left(\bar{S}_{1}\right),\left(\tilde{S}_{1}\right)\right)$ in [1], so that the Schrödinger operator could have the same good properties.

We denote $\left\{\lambda_{j}\right\}$ to be the eigenvalues sequence of $-\Delta+V(x)$ (see Proposition 2.1 in Section 2). Set $F(x, t)=\int_{0}^{t} f(x, s) d s, \mathcal{F}(x, t)=f(x, t) t-2 F(x, t)$.

We assume the following conditions.

$\left(f_{1}\right) f: \mathbb{R}^{N} \times \mathbb{R} \rightarrow \mathbb{R}$ is a Carathéodory function with a subcritical growth,

$$
|f(x, t)| \leq c\left(1+|t|^{s-1}\right), t \in \mathbb{R}, x \in \mathbb{R}^{N},
$$

where $s \in\left(2,2^{*}\right), f(x, t) \geq 0$ for all $(x, t) \in \mathbb{R}^{N} \times \mathbb{R}$ and $f(x, t)=o(|t|)$ as $|t| \rightarrow 0$.

$\left(f_{2}\right) \lim _{|t| \rightarrow+\infty} \frac{f(x, t) t}{|t|^{2}}=+\infty$ uniformly for $x \in \mathbb{R}^{N}$.

(c) 2011 Qian; licensee Springer. This is an Open Access article distributed under the terms of the Creative Commons Attribution License (http://creativecommons.org/licenses/by/2.0), which permits unrestricted use, distribution, and reproduction in any medium, provided the original work is properly cited. 
$\left(f_{3}\right)$ There exist $\theta \geq 1, s \in[0,1]$ s.t.

$$
\theta \mathcal{F}(x, t) \geq \mathcal{F}(x, s t),(x, t) \in \mathbb{R}^{N} \times \mathbb{R} .
$$

$\left(f_{4}\right) f(x, t)$ is odd in $t$.

Let us point out that, under our assumptions on $f(x, t)$, we can assume without loss of generality that $V$ is strictly positive just replacing $V(x)$ with $V(x)+L$ and $f(x, u)$ with $f(x, u)+L u, L$ large enough. We shall prove the following result.

Theorem 1.1 Under assumptions $\left(V_{1}\right),\left(V_{2}\right),\left(f_{1}\right)-\left(f_{4}\right)$, problem (1.1) has infinitely many sign-changing solutions.

Remark 1.1 In [2,3], they got sign-changing solutions for elliptic problem with Dirichlet boundary value. Those abstract results involved a Banach space of continuous functions in the Hilbert space, where the cone has a nonempty interior. This plays a crucial role. While the abstract theory in this paper only involved a Hilbert space, where the cone has an empty interior.

Remark 1.2 In [4], they showed infinitely many solutions for $p$-Laplace equation with Dirichlet boundary value, while we get infinitely many sign-changing solutions under similar conditions.

Remark 1.3 Equation 1.1 has been studied in [5], where they obtained the existence for sign-changing solutions in a asymptotically case.

Remark 1.4 In [1, \$5.3], they also obtained infinitely many sign-changing solutions for elliptic problem with Dirichlet boundary value, under (AR) condition stronger than $\left(f_{2}\right)$ and $\left(f_{3}\right)$ above.

Remark 1.5 In [1, \$6.4], Equation 1.1 has been studied the existence for infinitely many sign-changing solutions under conditions stronger than ours above.

\section{Preliminaries}

We consider the Hilbert space

$$
E=\left\{u \in H^{1}\left(\mathbb{R}^{N}\right): \int_{\mathbb{R}^{N}}\left(|\nabla u|^{2}+V(x) u^{2}\right) d x<\infty\right\}
$$

endowed with the inner product $(u, v)=\int_{\mathbb{R}^{N}}(\nabla u \nabla v+V(x) u v) d x$ for $u, v \in E$ and norm $\|u\|=(u, u)^{\frac{1}{2}}$. Clearly it is $E \leq H^{1}\left(\mathbb{R}^{N}\right)$. Denote $|u|_{q}$ to be the norm of $u$ in $L^{q}$ $\left(\mathbb{R}^{N}\right)$. In order to overcome the lack of compactness of the problem, the following proposition is crucial.

Proposition 2.1 [1,5] Assume $V(x)$ satisfies condition $\left(V_{1}\right)$ and $\left(V_{2}\right)$, or $\left(S_{1}\right)$ or $\left(\bar{S}_{1}\right)$ and $\left(\tilde{S}_{1}\right)$ in [1]. Then the imbedding $E \leqslant L^{q}\left(\mathbb{R}^{N}\right)$ is continuous if $q \in\left[2,2^{*}\right]$ and compact if $q \in\left[2,2^{*}[\right.$. Hence, the eigenvalue problem

$$
-\Delta u+V(x) u=\lambda u, \quad x \in \mathbb{R}^{N}
$$

possesses a sequence of positive eigenvalue

$$
0<\lambda_{1}<\lambda_{3}<\cdots<\lambda_{k}<\cdots \rightarrow \infty
$$

with finite multiplicity for each $\lambda_{k}$. Moreover, the principle eigenvalue $\lambda_{1}$ is simple with a positive eigenfunction $\phi_{1}$, and the eigenfunctions $\phi_{k}$ corresponding to $\lambda_{k}, k \geq 2$ are sign changing. 
Let us consider the functional $J: E \rightarrow \mathbb{R}$

$$
J(u)=\frac{1}{2}\|u\|^{2}-\int_{\mathbb{R}^{N}} F(x, u) d x .
$$

Then $J \in C^{1}(E, \mathbb{R})$ and $J^{\prime}=i d(-\Delta+V)^{-1} f=i d-K_{J}$. The critical point of $J$ is just the weak solution of problem (1.1).

The proof if our main results will be obtained by a suitable applications of an abstract critical point theorem stated in [1]. For completeness, we recall here this theorem.

Let $E$ be Hilbert space with norm $\|u\|$, and $Y, M$ be two subspaces of $E$ with $\operatorname{dim} Y$ $<\infty, \operatorname{dim} Y-\operatorname{co} \operatorname{dim} M \geq 1$. Let $G$ be $C^{1}$ - functional on $E$ with $G^{\prime}(u)=u-K_{G}(u)$ and $P$ denote a closed convex positive cone of $E$. Denote $\pm D_{0}$ by open convex subsets of $E$, containing the positive cone $P$ in its interior and $K=\left\{u \in E: G^{\prime}(u)=0\right\}, K[a, b]=\{u$ $\in K: G(u) \in[a, b]\}$. Set $D=D_{0} \cup\left(-D_{0}\right), S=E \backslash D$. In applications, $D$ contains all positive and negative critical points, and $S$ includes all possible sign-changing critical points. Hence, nontrivial sign-changing solutions can be obtained by different choose of $\pm D_{0}$ and $S$.

Next, we assume that there is another norm $\|\cdot\|_{*}$ of $E$ such that $\|u\|_{*} \leq c_{*}\|u\|$ for all $u \in E$, where $c_{*}>0$ is a constant. Moreover, we assume that $\left\|u_{n}-u\right\|_{*} \rightarrow 0$ whenever $u_{n} \rightarrow u$ weakly in $(E,\|\cdot\|)$. Write $E=M_{1} \oplus M$.

Let

$$
Q^{*}(\rho)=\left\{u \in M: \frac{\|u\|_{*}^{p}}{\|u\|^{2}}+\frac{\|u\|\|u\|_{*}}{\|u\|+D_{*}\|u\|_{*}}=\rho\right\}
$$

where $\rho>0, D_{*}>0, p>2$ are fixed constants. Let $Q^{* * *}=Q^{*}(\rho) \cap G^{\beta} \subset S$ and $\gamma=\inf _{Q * *} G$, where $G^{\beta}=\{u \in E: G(u) \leq \beta\}$, then $\beta \geq \gamma$.

Let us assume that

(A) $K_{G}\left( \pm D_{0}\right) \subset \pm D_{0}$;

$\left(A_{1}^{*}\right)$ Assume that for any $a, b>0$, there is a $c_{2}=c_{2}(a, b)>0$ such that $G(u) \leq a$ and $\|u\|_{*} \leq b \Rightarrow\|u\| \leq c_{2}$;

$\left(A_{2}^{*}\right) \lim _{u \in Y,\|u\| \rightarrow \infty}=-\infty, \sup _{Y} G=\beta$.

In the sequel, we shall consider the following Palais-Smale condition, shortly ( $w^{*}$ PS) condition.

Definition 2.1 The functional $G$ is said to satisfy the ( $w^{*}-$ PS) condition if any sequence $\left\{u_{n}\right\}$ such that $\left\{G\left(u_{n}\right)\right\}$ is bounded and $G^{\prime}\left(u_{n}\right) \rightarrow 0$, we have either $\left\{u_{n}\right\}$ is bounded and has a convergent subsequence or $\exists \sigma, R, \beta>0$ s.t. for any $u \in J^{1}([c-\sigma, c$ $+\sigma]$ ) with $\|u\| \geq R,\left\|J^{\prime}(u)\right\|\|u\| \geq \beta$. If in particular, $\left\{G\left(u_{n}\right)\right\} \rightarrow c$, we say that $\left(w^{*}-\right.$ $\mathrm{PS})_{c}$ is satisfied.

The following results hold (see [1, Theorem 5.6]).

Theorem 2.1 Assume $(A)$ and $\left(A_{1}^{*}\right)$ and $\left(A_{2}^{*}\right)$. If the even functional $G$ satisfies the $\left(w^{*}-\mathrm{PS}\right)_{c}$ condition at lever $c$ for each $c \in[r, \beta]$, then

$$
K[r-\varepsilon, \beta+\varepsilon] \cap(E \backslash P \cup(-P)) \neq \emptyset
$$

for all $\varepsilon>0$ small. 


\section{Proof of the main theorems}

From now on, we will denote by $N_{k}$ the eigenspace of $\lambda_{k}$. Then $\operatorname{dim} N_{k}<\infty$. We fix $k$ and let $E_{k}=N_{1} \oplus \ldots \oplus N_{k}$. In order to give the proof of Theorem 1.1, first we state some useful lemmas.

Lemma $3.1 J(u) \rightarrow-\infty$, as $\|u\| \rightarrow \infty$, for all $u \in E_{k}$.

Proof. Because $\operatorname{dim} E_{k}<\infty$, all norms in it are equivalent, then by $\left(f_{2}\right)$,

$$
\frac{J(u)}{\|u\|^{2}} \leq \frac{1}{2}-\int_{\mathbb{R}^{N}} \frac{F(x, u)}{\|u\|^{2}} d x \rightarrow-\infty .
$$

Consider another norm $\|\cdot\|::=\|\cdot\|_{s}$ of $E, s \in\left(2,2^{*}\right)$. Then $\|u\|_{s} \leq C_{*}\|u\|$ for all $u$ $\in E$, here $C_{*}>0$ is a constant and by lemma $2.1\left\|u_{n}-u\right\|_{*} \rightarrow 0$ whenever $u_{n} \rightarrow u$ weakly in $E$. Write $E=E_{k-1} \oplus E_{k-1}^{\perp}$. Let

$$
Q^{*}(\rho)=\left\{u \in E_{k-1}^{\perp}: \frac{\|u\|_{s}^{s}}{\|u\|^{2}}+\frac{\|u\|\|u\|_{s}}{\|u\|+D_{*}\|u\|_{s}}=\rho\right\}
$$

where $\rho, D^{*}$ are fixed constants.

Lemma 3.2 $\|u\|_{s} \leq c_{1}, \forall u \in Q^{*}(\rho)$, where $c_{1}>0$ is a constant.

Proof. If $\|u\|_{s} \rightarrow \infty$, then so does $\|u\| \rightarrow \infty$. Hence

$$
\frac{\|u\|\|u\|_{s}}{\|u\|+D_{*}\|u\|_{s}} \rightarrow \infty
$$

a contradiction.

By $\left(f_{1}\right)$, there exist $C_{F}>0, s \in\left(2,2^{*}\right)$ such that

$$
|F(x, u)| \leq \frac{\lambda_{1}}{4} u^{2}+C_{F}|u|^{s}, \quad x \in \mathbb{R}^{N}, u \in \mathbb{R} .
$$

Therefore, for any $a, b>0$, there is a $c_{2}=c_{2}(a, b)>0$ such that

$$
J(u) \leq a,\|u\|_{s} \leq b \Rightarrow\|u\| \leq c_{2} .
$$

By lemma 3.1,

$$
\lim _{u \in Y,\|u\| \rightarrow \infty} J(u)=-\infty,
$$

where $Y=E_{k}$. Then, conditions $\left(A_{1}^{*}\right)$ and $\left(A_{2}^{*}\right)$ are satisfied. We define

$$
\sup _{Y} G:=\beta \text {. }
$$

Let

$$
Q^{* *}:=Q^{*}(\rho) \cap J^{\beta} \subset S, \quad \inf _{Q^{* *}}:=\gamma .
$$

Set $P=\left\{u \in E: u(x) \geq 0\right.$ for a.e. $\left.x \in \mathbb{R}^{N}\right\}$. Then, $P(-P)$ is the positive (negative) cone of $E$ and weakly closed. By Lemma 5.4 or Lemma 6.8 [1], there is a $\delta:=\delta(\beta)$ such that $\operatorname{dist}\left(Q^{* *}, P\right)=\delta(\beta)>0$. We define

$$
D\left(\mu_{0}\right):=\left\{u \in E: \operatorname{dist}(u, P)<\mu_{0}\right\},
$$

where $\mu_{0}$ us determined by the following lemma.

Lemma 3.3 Under the assumptions $\left(V_{1}\right),\left(V_{2}\right)$, and $\left(f_{1}\right)$, there is a $\mu_{0} \in(0, \delta)$ (may be chosen small enough) such that $K_{J}\left( \pm D\left(\mu_{0}\right)\right) \subset \pm D\left(\mu_{0}\right)$. Therefore, $(A)$ is satisfied. 
Proof. Please see Lemma 2.9 of [1] for the similar proof.

Let $D:=-D\left(\mu_{0}\right) \cup D\left(\mu_{0}\right), S:=E \backslash D$. By Lemma 3.3, we may assume $Q^{* *} \subset S$.

Lemma 3.4 Let us assume that $\left(V_{1}\right),\left(V_{2}\right)$ and $\left(f_{2}\right),\left(f_{3}\right)$ hold. Then, the functional $J$ satisfies the ( $w^{*}$-PS) condition.

Proof. As the sequence $\left\{u_{n}\right\}$ such that $\left\{G\left(u_{n}\right)\right\}$ is bounded and $G^{\prime}\left(u_{n}\right) \rightarrow 0$, if $\left\{u_{n}\right\}$ is bounded, then by Proposition 2.1 and the compact imbedding $E \leqslant L^{q}\left(\mathbb{R}^{N}\right), q \in\left[2,2^{*}[\right.$, we have $\left\{u_{n}\right\}$ possesses a convergent subsequence.

Next to prove another case. If not, there exist $c \in \mathbb{R}$ and $\left\{u_{n}\right\} \subset E$ satisfying, as $n \rightarrow$ $\infty$

$$
J\left(u_{n}\right) \rightarrow c,\left\|u_{n}\right\| \rightarrow \infty,\left\|J^{\prime}\left(u_{n}\right)\right\|\left\|u_{n}\right\| \rightarrow 0
$$

then we have

$$
\begin{aligned}
& \lim _{n \rightarrow \infty} \int_{\mathbb{R}^{N}}\left(\frac{1}{2} f\left(x, u_{n}\right) u_{n}-F\left(x, u_{n}\right)\right) d x \\
= & \lim _{n \rightarrow \infty}\left(J\left(u_{n}\right)-\frac{1}{2}<J^{\prime}\left(u_{n}\right), u_{n}>\right)=c .
\end{aligned}
$$

Denote $v_{n}=\frac{u_{n}}{\left\|u_{n}\right\|}$, then $\left\|v_{n}\right\|=1$, that is $\left\{v_{n}\right\}$ is bounded in $E$. Thus, up to a subsequence, for some $v \in E$, we get

$$
\begin{gathered}
v_{n} \rightarrow \text { vin } E, \\
v_{n} \rightarrow \operatorname{vin} L^{p}\left(\mathbb{R}^{N}\right), \text { for } 2 \leq p<2^{*}, \\
v_{n}(x) \rightarrow v(x) \text { a.e. } x \in \mathbb{R}^{N} .
\end{gathered}
$$

If $v \neq 0$, because $\left\|J^{\prime}\left(u_{n}\right)\right\|\left\|u_{n}\right\| \rightarrow 0$, as the similar proof in Lemma 6.22 of [2] or Lemma 2.2 of [4], we get a contradiction.

If $v=0$, by condition $\left(f_{3}\right)$, as the similar proof in Lemma 6.22 of [2] or Lemma 2.2 of [4], we also have

$$
\int_{\mathbb{R}^{N}}\left(\frac{1}{2} f\left(x, u_{n}\right) u_{n}-F\left(x, u_{n}\right)\right) d x \rightarrow \infty,
$$

which contradicts (3.3).

This proves that $J$ satisfies the ( $w^{*}$-PS) condition.

Remark 3.1 Our condition $\left(f_{3}\right)$ here is different from $\left(P_{3}\right)$ of [1, Theorem 6.14 ], which is used to prove the ( $\left.\mathrm{w}^{*}-\mathrm{PS}\right)$ condition; furthermore, it is more weaker.

Proof of Theorem 1.1. By Theorem 2.1,

$$
K[r-\varepsilon, \beta+\varepsilon] \cap(E \backslash P \cup(-P)) \neq \varnothing
$$

for all $\varepsilon>0$ small. That is there exists a $u_{k} \in E \backslash(-P \cup P)$ (sign-changing critical point) such that

$$
J^{\prime}\left(u_{k}\right)=0, \quad J\left(u_{k}\right) \in[r-1, \beta+1] .
$$

Next, we estimate the $\gamma=\inf _{Q^{* *}}$. Because of Proposition 2.1, we can adopt the similar method as in [1, p. 67]. Similar to Lemma 2.23 of [1], by choosing the constants $D$ * and $\rho$, for all $u \in Q^{*}(\rho)$, we may get 


$$
\|u\| \geq \Lambda_{s}^{*} \min \left\{\lambda_{k}^{(1-\alpha)(s-2) / 2}, \lambda_{k}^{(1-\alpha) / 2}\right\} \min \left\{\rho, \rho^{1 /(s-2)}\right\} .
$$

By Lemma 2.26 of [1], for any $u \in Q^{*}(\rho)$, we have that

$$
J(u) \geq \frac{1}{8}\left(\Lambda_{s}^{*}\right)^{2} T_{1} T_{2},
$$

where $\Lambda_{s}^{*}, T_{1}, T_{2}$ are defined in (2.49)-(2.51) in [1] with $p$ replaced by $s \in\left(2,2^{*}\right), \alpha \in$ $(0,1)$ is a constant, and $\Lambda_{s}^{*}, T_{2}$ are independent of $k$. In particular, since $\lambda_{k} \rightarrow \infty$, we get

$$
T_{1}:=\min \left\{\lambda_{k}^{(1-\alpha)(s-2) / 2}, \lambda_{k}^{(1-\alpha) / 2}\right\} \rightarrow \infty, \text { as } k \rightarrow \infty .
$$

Therefore, $\gamma \rightarrow \infty$ as $k \rightarrow \infty$; hence the proof of Theorem 1.1 is finished.

\section{Acknowledgements}

The author thanks professor Wenming Zou for his encouragements. This study was supported by the Chinese National Science Foundation (10726003,11001151), the National Science Foundation of Shandong (Q2008A03) and the Science Foundation of China Postdoctoral(201000481301) and Shandong Postdoctoral.

\section{Competing interests}

The author declares that they have no competing interests.

Received: 2 March 2011 Accepted: 3 October 2011 Published: 3 October 2011

\section{References}

1. Zou, WM: Sign-Changing Critical Point Theory. Springer (2008)

2. Li, SJ, Wang, ZQ: Ljusternik-Schnirelman theory in partially ordered Hilbert spaces. Trans Am Math Soc. 354, 3207-3227 (2002). doi:10.1090/S0002-9947-02-03031-3

3. Qian, AX, Li, SJ: Multiple nodal solutions for elliptic equations. Nonlinear Anal T.M.A. 57, 615-632 (2004). doi:10.1016/j. na.2004.03.010

4. Liu, SB, Li, SJ: Infinitely many solutions for a super elliptic equation. Acta Mathematica Sinica Chinese Series. 46 , 625-630 (2003)

5. Salvatore, A: Sign-changing solutions for an asympotically linear Schrödinger equation. Discrete Contin Dyn Syst Suppl. 669-677 (2009)

\section{Submit your manuscript to a SpringerOpen ${ }^{\circ}$} journal and benefit from:

Convenient online submission

Rigorous peer review

- Immediate publication on acceptance

- Open access: articles freely available online

- High visibility within the field

- Retaining the copyright to your article

Submit your next manuscript at $\mathbf{s p r i n g e r o p e n . c o m ~}$ 